\title{
A Method for the Numerical Evaluation of the Second Virial Coefficient for Polyatomic Molecules
}

\author{
P. M. Holland \\ Department of Chemistry, University of Colorado, Boulder, Colorado 80302
}

J. F. Ely*

Department of Chemical Engineering, Rice University, Houston, Texas 77001

\author{
H. J. M. Hanley**
}

Institute for Basic Standards, National Bureau of Standards, Boulder, Colorado 80302

and

Department of Chemical Engineering, University of Colorado, Boulder, Colorado 80302

(June 29, 1977)

\begin{abstract}
A numerical integration procedure to calculate the second virial coefficient of simple polyatomic molecules is proposed. The intermolecular pair potential is assumed to be a sum of a spherically symmetric contribution and an unsymmetric, angular dependent, contribution. The method is based on evaluating the possible different numerical values for this latter term. Quadrupolar and dipolar molecules are considered. Calculations for the virial coefficient for quadrupolar molecules are judged to be correct to within one part in 2500 or better, and to within one part in 300 or better for polar molecules. Results from the method are compared to corresponding results from the well-known Pople expansion procedure. It is shown that care must be taken to ensure this latter technique yields a convergent answer.
\end{abstract}

Key words: Dipolar gas; numerical integration; polyatomic molecule; Pople expansion; quadrupolar gas; second virial coefficient.

\section{Introduction}

In several papers $[1,2,3],{ }^{1}$ we have determined the thermophysical properties of simple polyatomic gases via statistical mechanics. In particular, a modification of the Pople expansion procedure [4] was used to evaluate the second virial coefficient. This technique is standard but there are some drawbacks when it is applied to molecules with relatively large electrostatic moments. The object of this paper is to introduce an alternative procedure.

The second virial coefficient, $B$, is defined by the expansion

$$
\frac{p}{\rho R T}=1+B \rho+\ldots
$$

(where $p$ is the pressure, $T$ the temperature, $R$ the gas constant and $\rho$ the density $(\mathrm{mol} / \mathrm{L}))$ and is given by an expression of the form

$$
B \sim N \iint\left\{\exp \left[-\beta \phi\left(\mathbf{R}_{2}, \mathbf{R}_{2}\right)\right]-1\right\} d \mathbf{R}_{1} d \mathbf{R}_{2}
$$

${ }^{1}$ Figures in brackets indicate the literature references at the end of this paper.
}

where $N$ is Avogadro's number, $\beta=1 / k T$ ( $k$ is Boltzmann's constant), $\phi$ is the pair potential, and $\mathbf{R}_{1}$ and $\mathbf{R}_{2}$ denote the position and angular configuration of molecule 1 and 2 , respectively.

In this work the intermolecular interaction of two polyatomic molecules is considered in the simple, but standard coordinate system of figure 1 : the molecules are treated as

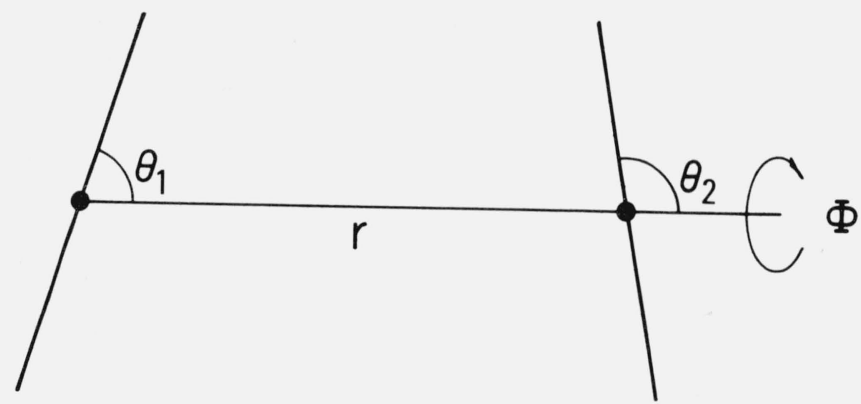

FigurE 1. The coordinate system for two polyatomic molecules.

rigid bodies interacting along the line of their centers, distance $r$ apart, with a relative orientation given by the angles $\theta_{1}, \theta_{2}$ and $\Phi$. The potential can be written as a sum: 


$$
\phi^{*}\left(r^{*}, \theta_{1}, \theta_{2}, \Phi\right)=\theta_{s}^{*}\left(r^{*}\right)+\theta_{n s}^{*}\left(r^{*}, \theta_{1}, \theta_{2}, \Phi\right)
$$

with $\phi_{s}^{*}\left(r^{*}\right)$ the spherically symmetric, angular independent, part and $\phi_{n s}^{*}$ the unsymmetric angular dependent part. In eq (3), $r^{*}=r / \sigma$ and $\phi^{*}=\phi / \epsilon$, where $\sigma$ and $\epsilon$ are a length and energy parameter of the spherical potential, respectively (to be defined below).

For the coordinate system of figure 1 and the potential of eq (3), eq (2) becomes

$$
\begin{aligned}
B^{*}= & B / b_{0}= \\
& -3 /(8 \pi) \int_{0}^{\pi} \sin \theta_{1} d \theta_{1} \int_{0}^{\pi} \sin \theta_{2} d \theta_{2} \int_{0}^{2 \pi} d \Phi \\
& \times \int_{0}^{\infty}\left\{\exp \left[-\phi^{*}\left(r^{*}, \theta_{1}, \theta_{2}, \Phi\right) / T^{*}\right]-1\right\} r^{* 2} d r^{*}
\end{aligned}
$$

where $b_{0}=(2 / 3) \pi N \sigma^{3}$ and $T^{*}=T /(\epsilon / k)$.

In previous studies [1-3], we have taken $\phi_{s}^{*}$ to be the $m$ 6-8 function of Klein and Hanley [5]

$$
\begin{aligned}
\phi_{s}^{*}\left(r^{*}\right)= & \frac{6+2 \gamma}{m-6}\left(\frac{\sigma}{r}\right)^{m} d^{m} \\
& -\frac{m-\gamma(m-8)}{m-6}\left(\frac{\sigma}{r}\right)^{6} d^{6}-\gamma\left(\frac{\sigma}{r}\right)^{8} d^{8}
\end{aligned}
$$

where $d=r_{\min } / \sigma$, with $\sigma$ and $r_{\min }$, and also the energy $\boldsymbol{\epsilon}$, defined by the conditions $\phi(\sigma)=0$ and $\phi\left(r_{\min }\right)=-\epsilon$. The parameters $m$ and $\gamma$ represent the "hardness" of the repulsive term, and the "strength" of the inverse eight attractive term, respectively.

If we consider non-polarizable axially symmetric quadrupolar molecules, $\phi_{n s}^{*}$ is given by

$$
\phi_{n s}^{*}\left(r^{*}, \theta_{1}, \theta_{2}, \Phi\right)=\frac{3}{4} \frac{\Theta^{* 2}}{r^{* 5}} f\left(\theta_{1}, \theta_{2}, \Phi\right)
$$

where

$$
\begin{aligned}
& f\left(\theta_{1}, \theta_{2}, \Phi\right)= \\
& 1-5 \cos ^{2} \theta_{1}-5 \cos ^{2} \theta_{2}-15 \cos ^{2} \theta_{1} \cos ^{2} \theta_{2} \\
& \quad+2\left(\sin \theta_{1} \sin \theta_{2} \cos \Phi-4 \cos \theta_{1} \cos \theta_{2}\right)
\end{aligned}
$$

and $\theta^{* 2}$ is the reduced quadrupole moment (squared); $\theta^{* 2}$ $=\theta^{2} /\left(\epsilon \sigma^{5}\right)$.

To be consistent with our earlier calculations, and those of other authors, we will first consider the potential given by eqs (3), (5), and (6) but with $\gamma=0$ and $m=12$ for which eq (5) becomes the Lennard-Jones, 12-6, potential.

\section{Procedure}

The evaluation of the second virial is carried out in four steps as follows. First, the quantity $f\left(\theta_{1}, \boldsymbol{\theta}_{2}, \Phi\right)$ of eq (7) is considered. Specifically, the molecules are set in a selected initial configuration, e.g., $\theta_{1}=\theta_{2}=\Phi=0$, then each of the angles is varied incrementally (e.g., by $\pi / 4$ ) and the numerical value of $f$ noted after each incremental step. Because of symmetry, many of the values are identical and it is only necessary to record, say, $l$ different values $f_{i}(i=$

\begin{tabular}{|c|c|c|c|}
\hline \multirow[t]{2}{*}{$\begin{array}{l}\text { Incremental } \\
\text { step } \pi / n\end{array}$} & \multicolumn{2}{|c|}{$\begin{array}{l}\text { Number of different }(l) \text { values for } \\
\text { the angle function at two selected } \\
\text { initial configurations: } C_{0} \text { and } C_{\pi / 2 n}\end{array}$} & \multirow{2}{*}{$\begin{array}{l}\text { Number of } \\
\text { possible values } \\
\text { for the angle } \\
\text { function }\end{array}$} \\
\hline & $\mathrm{C}_{0}$ & $\mathrm{C}_{\pi / 2 n}$ & \\
\hline \multicolumn{4}{|c|}{ (a) Quadrupolar } \\
\hline$\pi / 4$ & 11 & 12 & 128 \\
\hline$\pi / 8$ & 74 & 80 & 1024 \\
\hline$\pi / 16$ & 548 & 576 & 8192 \\
\hline \multicolumn{4}{|c|}{ (b) Dipolar } \\
\hline$\pi / 4$ & 13 & 24 & 128 \\
\hline$\pi / 8$ & 123 & 160 & 1024 \\
\hline$\pi / 16$ & 1011 & 1152 & 8192 \\
\hline
\end{tabular}
$1, l)$, and their number of occurrences or weight, $\mathrm{wt}_{i}$. In general $f_{i}$ and its weight, $w_{i}$, are associated with the initial configuration $C_{x}^{\prime}$ and the step $\pi / n$ where $x \equiv 0$ or $\pi / 2 n$ with $n=2,4,8 \ldots$. That the number $l$ is relatively small compared to the total number of configurations is shown in table 1 .

TABLE 1. Values of $l$ for various incremental steps and initial configurations of two molecules in the coordinate system of figure 1 . (a) Quadrupolar interactions, (b) dipolar interactions.

Second, an intergral $I_{i}$ is defined by

$$
I_{i}=\int_{0}^{\infty} \exp \left[-\phi_{i}^{*} / T^{*}-1\right] r^{* 2} d r^{*} \quad i=1, l
$$

to correspond to the $r^{*}$ integral in eq (4). Here $\phi_{i}{ }^{*}$ is the potential of eq (3) for a given quadrupole moment associated with a particular $f_{i}\left(\theta_{1}, \theta_{2}, \Phi\right)$.

The integral is evaluated numerically at a selected $T^{*}$ by the one-dimensional Chebyshev integration technique used in our previous work [1,2].

Third, the value of the reduced second virial coefficient for an initial configuration, $C_{x}$, and an incremental step follows from the expression

$$
B^{*}\left(T^{*}, \Theta^{* 2}\right)=-3 \frac{\sum_{i=1}^{l} \Omega_{i} I_{i}}{\sum_{i=1}^{l} \Omega_{i}}
$$

where

$$
\Omega_{i}=\left(\sin \theta_{1} \sin \theta_{2} \mathrm{wt}\right)_{i} \quad i=1, l .
$$

Fourth, and last, since $B^{*}$ of eq (9) is associated with $C_{x}$ and the incremental step, a final estimate of the virial coefficient is obtained from the results for different steps at a particular $C_{x}$, and then from a repeat of the procedure for a different $C_{\boldsymbol{x}}$.

\subsection{Variation of $I_{i}$ With the Angle Function}

The calculation outlined above is potentially time-consuming, despite the simplification due to symmetry, because the integral $I_{i}$ apparently needs to be evaluated $l$ times for each step at each configuration. We have, however, observed a feature which allows the calculation to be manageable: 
namely, that $I_{i}$ appears to vary smoothly with the angle function at a given $T^{*}$ and $\Theta^{* 2}$. This is illustrated by figure 2 in which $3 I_{i}$ is plotted versus $f_{i}\left(\theta_{1}, \theta_{2}, \Phi\right)$ for the $12-6$ potential with $\boldsymbol{\theta}^{* 2}=0.7$ at three representative temperatures. Hence for a given spherical potential with a given $\boldsymbol{\Theta}^{* 2}$ at $T^{*}$, one only has to evaluate a few $I_{i}$ directly: the remainder can be estimated to a high precision by interpolation.

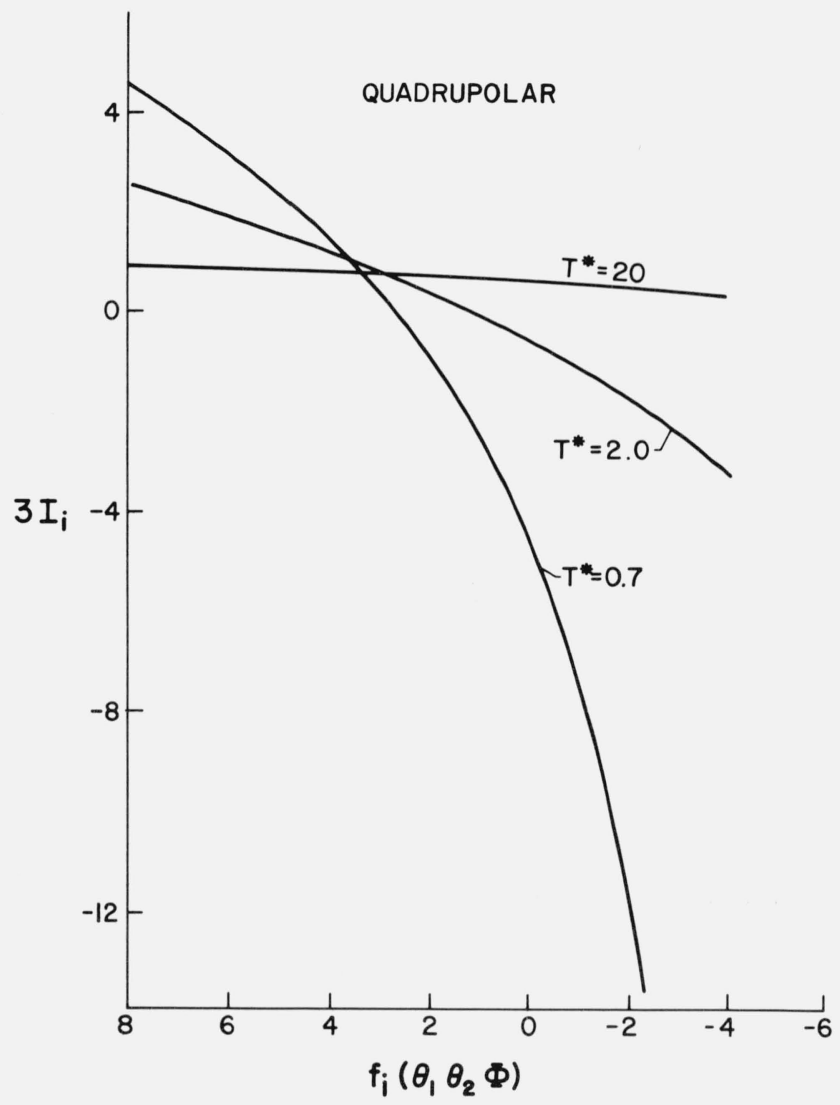

Figure 2. Plot of $3 I_{i}$ eq (8), versus $\mathrm{f}_{\mathrm{i}}\left(\boldsymbol{\theta}_{1} \boldsymbol{\theta}_{2} \Phi\right)$, from eq (7), at $T^{*}=0.7$, 2.0, and 20.0 for a 12-6 quadrupolar molecule with $\theta^{* 2}=0.7$.

\section{Results}

\subsection{Quadrupolar Molecules}

Sample detailed results are presented in table 2 which displays values of $B^{*}$ for several incremental steps and initial configurations at four reduced temperatures; $0.7,1.0,2.0$, and 20.0. The spherical potential was taken to be the 12-6 and the reduced quadrupole moment (squared) was set at 0.2 -which corresponds to the moment (squared) for a molecule similar to nitrogen. Our estimated convergent values are also shown and are judged to be precise to within one part in 2500 at $T^{*}=0.7$ and to one part in 10,000 at $T^{*}=20.0$.

A check on the absolute values of the virials listed in table 2 was made by comparing them to corresponding values from the expansion technique [4]: the expansion procedure can be expected to yield essentially an exact result for $B^{*}$ provided the quadrupole moment is small. For the potential of eq (3) with equations (5) and (6) one can derive $[2,6]$ :

$$
\begin{aligned}
B^{*}\left(T^{*}\right)=B_{s}^{*}-\frac{21}{5} \frac{\Theta^{* 4}}{T^{* 2}} J_{10} & +\frac{216}{245} \frac{\Theta^{* 6}}{T^{* 3}} J_{15}-\frac{639}{245} \frac{\Theta^{* 8}}{T^{* 4}} J_{20} \\
& +\frac{4608}{4235} \frac{\Theta^{* 10}}{T^{* 5}} J_{28}+\cdots
\end{aligned}
$$

TABLE 2. The second virial coefficient of a quadrupolar molecule, $\theta^{* 2}=$ 0.2 , as a function of initial configuration and incremental step (see text).

\begin{tabular}{|c|c|c|c|c|c|}
\hline \multirow{2}{*}{$T^{*}$} & \multirow{2}{*}{$\begin{array}{c}\text { Incre- } \\
\text { mental } \\
\text { step } \\
(\pi / n)\end{array}$} & \multicolumn{2}{|c|}{ Initial configuration } & \multirow{2}{*}{$\begin{array}{l}\text { Estimated* } \\
\text { convergent } \\
\text { value }\end{array}$} & \multirow{2}{*}{$\begin{array}{l}\text { Equation } \\
\text { (11) }\end{array}$} \\
\hline & & $\mathrm{C}_{0}$ & $\mathrm{C}_{\pi / 2 n}$ & & \\
\hline 0.7 & $\begin{array}{l}\pi / 4 \\
\pi / 8 \\
\pi / 16\end{array}$ & $\begin{array}{l}-4.80711 \\
-4.84904 \\
-4.85425\end{array}$ & $\begin{array}{l}-4.86570 \\
-4.85841 \\
-4.85637\end{array}$ & -4.8557 & -4.8556 \\
\hline 1.0 & $\begin{array}{l}\pi / 4 \\
\pi / 8 \\
\pi / 16\end{array}$ & $\begin{array}{l}-2.57073 \\
-2.58929 \\
-2.59140\end{array}$ & $\begin{array}{l}-2.59522 \\
-2.59293 \\
-2.59220\end{array}$ & -2.5919 & -2.5919 \\
\hline 2.0 & $\begin{array}{l}\pi / 4 \\
\pi / 8 \\
\pi / 16\end{array}$ & $\begin{array}{l}-.63202 \\
-.63775 \\
-.63828\end{array}$ & $\begin{array}{l}-.63862 \\
-.63857 \\
-.63845\end{array}$ & -0.63840 & -0.63834 \\
\hline 20.0 & $\begin{array}{l}\pi / 4 \\
\pi / 8 \\
\pi / 16\end{array}$ & $\begin{array}{l}.52567 \\
.52522 \\
.525193\end{array}$ & $\begin{array}{l}.52534 \\
.52519 \\
.525189\end{array}$ & .525190 & .525185 \\
\hline
\end{tabular}
Comparisons with equivalent values from the expansion of equation (11) are given.

* Estimated by plotting $B^{*}$ versus $\pi / n$ for both $\mathrm{C}_{0}$ and $\mathrm{C}_{\pi / 2 n}$. The intersection point of the two curves was taken as the convergent value.

where $B_{s}^{*}$ is the angular-independent, classical second virial at $T^{*} . J_{x}$ are dimensionless integrals given by

$$
J_{x}=\int_{0}^{\infty} \exp \left[-\phi_{s}^{*}\left(r^{*}\right) / T^{*}\right] r^{*(-x+2)} d r^{*} .
$$

Results from eq (11), for the $12-6$ potential and $\Theta^{* 2}=$ 0.2 , are given in the last column of table 2. Since the agreement between corresponding second virial coefficients from our procedure and from equation (11) is very close, we conclude our procedure is satisfactory.

\subsection{Polar Molecules}

The integration procedure is not, of course, restricted to quadrupolar molecules. It was straightforward to extend it to polar molecules interacting with a potential of the form

$$
\phi^{*}\left(r^{*}, \theta_{1}, \theta_{2}, \phi\right)=\theta_{s}^{*}\left(r^{*}\right)-\frac{\mu^{* 2}}{r^{* 3}} g\left(\theta_{1}, \theta_{2}, \Phi\right)
$$

where

$$
g\left(\theta_{1}, \theta_{2}, \Phi\right)=2 \cos \theta_{1} \cos \theta_{2}-\sin \theta_{1} \sin \theta_{2} \cos \Phi .
$$


$\mu^{*}$ is the reduced dipole moment given by $\mu^{* 2}=\mu /\left(\epsilon \sigma^{3}\right)$, where $\mu$ is the dipole moment. $\phi_{s}^{*}\left(\tau^{*}\right)$ is the spherical potential of eq (5), as before.

Calculations followed those for quadrupolar molecules using the two significant time-saving arguments, i.e., (a) the symmetry of the coordinate systems which reduces substantially the number of independent values of $g\left(\theta_{1}, \theta_{2}, \Phi\right)$ which have to be considered (see table 1), and (b) that a particular value of $g, g_{i}$, is a smooth function of the $r$ integral, $I_{i}$ [ eq (8)], see figure 3 . Typical results are shown in table 3 for three temperatures. The results are those for a 12-6 potential for $\mu^{* 2}=0.8485$ and 2.8284 , respectively. We have listed the parameter $\tau^{*}$, used in Hirschfelder, Curtiss, and Bird [7] and elsewhere [ $\left.\tau^{*}=8^{-1 / 2} \mu^{* 2}\right]$. For these dipole moments (squared), $\tau^{*}=0.3$ and 1.0 .

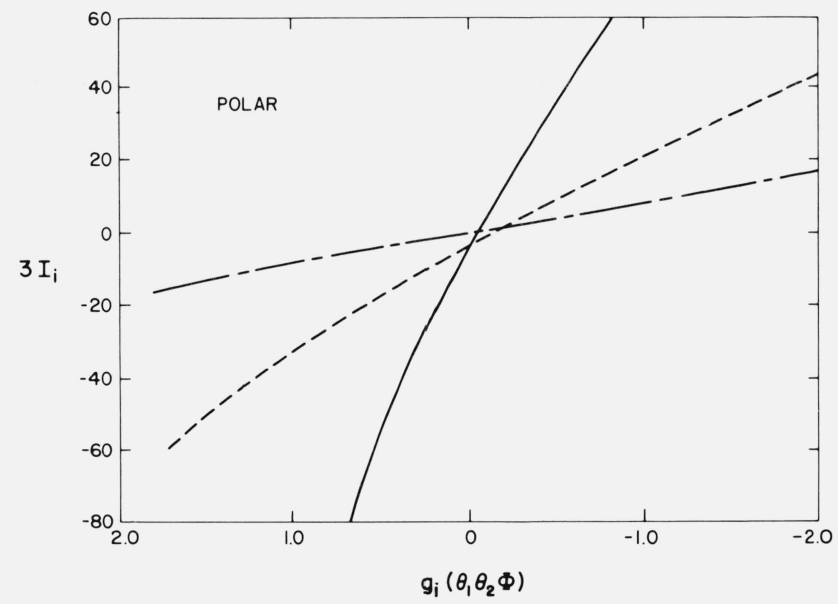

Figure 3. Plot of $3 I_{i}$ eq $(8)$, versus $\mathrm{g}_{(}\left(\theta_{1} \theta_{2} \Phi\right)$, from eq (14), at $T^{*}=$ 0.7 and $\tau^{*}=1.0$ (solid line), $T^{*}=0.7$ and $\tau^{*}=0.3$, (dashed line) and $T^{*}=2.0$ and $\tau^{*}=0.3$ (dotted line).

TABLE 3. Comparison between the sound virial coefficient for a polar molecule calculated by numerical integration, and by the expansion of equation (15)

\begin{tabular}{rcc}
\hline \hline$T^{*}$ & $B^{*}$, integration & $B^{*}$, expansion \\
\hline \multicolumn{3}{c}{$\tau^{*}=0.3$} \\
2.0 & -6.001 & -6.0182 \\
20.0 & -0.7130 & -0.7168 \\
& .5241 & .52441 \\
\hline \multicolumn{3}{c}{$\tau^{*}=1.0$} \\
2.0 & -41.61 & -39.89 \\
20.0 & -1.706 & -1.7264 \\
& .5148 & .51468 \\
\hline
\end{tabular}

Again our results can be compared to those from the expansion technique [6]:

$$
=B_{s}^{*}-192 \sum_{j=1}^{\infty}\left[\frac{B^{*}\left(T^{*}\right)}{(2 j+1) !}\right]^{2} \mu^{* 4 j} K_{j} J_{6 j}\left[\frac{2^{2(j-3)}}{T^{* 2 j}}\right]
$$

where

$$
K_{j}=\sum_{k=0}^{j}(2 k) ! /\left(k^{\prime}\right)^{2}
$$

and $J_{6 J} \equiv J_{x}$ of eq (12). Values of $B^{*}$ from eq (15) for $j=4$ are given in table 3 .
The results from our integration procedure are apparently low. But this is most probably due to the appreciable contribution of the term $\left(\mu^{* 2} / r^{3}\right) g\left(\theta_{1}, \theta_{2}, \Phi\right)$ to the integral eq (8) at large values of $r$. Our Chebyshev integration method can fail to yield a fully convergent answer in such cases. Clearly, one could compensate for this difficulty. However, the substantial discrepancy at $T^{*}=0.7$ for $\tau^{*}=$ 1.0 is undoubtedly a failure of the expansion (15) to give a convergent for $j=4$.

\subsection{Mixed Moments}

The method can be applied to molecules with more than one electrostatic moment and, in fact, has been used for nitrous oxide: a molecule which has a small dipole and a large quadrupole [3]. We considered the potential

$$
\phi^{*}\left(r^{*}, \theta_{1}, \theta_{2}, \Phi\right)=\phi_{s}^{*}+\phi_{D D}^{*}+\phi_{Q Q}^{*}+\phi_{Q D}^{*}
$$

where $\phi_{s}^{*}$ is eq $(5) ; \phi_{D D}^{*}$ is the angle dependent term of eq (13); $\phi_{Q Q}^{*}$ is given by eq (6); while $\theta_{Q D}^{*}$, the contribution of the quadrupole-dipole interaction, is given by

$$
\begin{aligned}
\phi_{Q D}^{*}= & \frac{3}{2 r^{* 4}} \mu^{*} \Theta^{*}\left[( \operatorname { c o s } \theta _ { 2 } - \operatorname { c o s } \theta _ { 1 } ) \left(3 \cos \theta_{1} \cos \theta_{2}\right.\right. \\
& \left.\left.-2 \sin \theta_{1} \sin \theta_{2} \cos \Phi\right)-\cos \theta_{1}+\cos \theta_{2}\right] .
\end{aligned}
$$

We will not report here results for the second virial coefficient of nitrous oxide; suffice to say that agreement between our method and that from the corresponding expansion [6] was within the precision of the respective determinations.

\section{Discussion and Conclusions}

We have introduced a simple numerical procedure to evaluate the second virial coefficient of a polyatomic molecule assuming the electrostatic moments are placed at the center of the molecule.

Our method should be compared, in general, to the expansion procedure since both techniques are based on the same approximations of the nonspherical intermolecular interaction. The expansion procedure treats the angle dependent terms of the potential as perturbations around the spherical contribution. Hence one obtains an expansion involving products of integrals of the spherical contribution and the electrostatic moments. For example, we have used eq (11) with the $J_{x}$ integrals. The advantage of this approach is that the $J_{x}$ integrals need only to be calculated once for a given spherical potential and one has some flexibility in adding various angle dependent terms.

In contrast, our method has the advantage that the $l$ different angle functions and their weights [e.g., eq (7) and eq (10)] only have to be recorded once for any type of angle dependence in the potential. The method, therefore, is flexible as to the spherical part of the potential.

Obviously, this flexibility has to be balanced against the problem that several $J_{x}$ integrals [eq (8)] have to be evaluated at a given $T^{*}$. Overall, therefore, the expansion procedure is faster. However, we have shown [1, 2] that calculations of the thermophysical properties in general, and of the second virial coefficient, in particular, for a gas are about as 
sensitive to a choice of the spherical part of the potential as they are to the various nonspherical contributions. The net time required, therefore, to calculate a value of $B^{*}$ for a given gas, is not too dependent on which of the two methods is used.

When comparing the two methods one assumes that the expansion procedure can yield a convergent result for the number of terms considered. But this is known to be a problem for polar molecules. In fact we have pointed out that, for the sample calculation at $T^{*}=0.7, \tau^{*}=1.0$ (table 3), the expansion of equation (15) has not given a convergent $B^{*}$ if $j=4$. It is less appreciated that convergence should also be checked for quadrupolar molecules, as was mentioned with respect to carbon dioxide in reference [2]. [Inspection of the expansion of equation (11) indicates one should anticipate difficulties if, by chance, the factor $\Theta^{* 2} / T^{*}$ is close to unity.]

As a matter of interest, we checked the convergence of the expansion of eq (15) for a molecule with a reduced quadrupole moment (squared) of 0.7 (to correspond approximately with carbon dioxide). The reduced second virial coefficients from our numerical procedure were taken to be correct. Table 4 lists $B^{*}$ (numerical) at four temperatures

TABLE 4. Comparison between values of $B^{*}$ for a quadrupolar molecule with $\theta^{* 2}=0.7$ calculated numerically, and calculated from the expansion of equation (11) to various orders in $1 / T^{*}$

\begin{tabular}{rcccc}
\hline \hline$T^{*}$ & $B^{*}$ & \multicolumn{3}{c}{$B^{*}[$ equation $(11)]$} \\
\hline & (numerical) & $0\left(1 / T^{* 3}\right)$ & $0\left(1 / T^{* 4}\right)$ & $0\left(1 / T^{* 5}\right)$ \\
\hline 0.7 & -6.718 & -6.2941 & -6.7729 & -6.6173 \\
1.0 & -3.232 & -3.1461 & -3.2434 & -3.2194 \\
2.0 & -.7599 & -0.7540 & -0.7605 & -0.7596 \\
20.0 & .5231 & .52313 & .52312 & .52312 \\
\hline
\end{tabular}

and the value of $B^{*}$ calculated from the expansion of eq (11) to order $1 / T^{* 3}, 1 / T^{* 4}$, and $1 / T^{* 5}$, respectively. The results suggest that the expansion technique can fail to give a convergent answer at $T^{*}=0.7$ (and possibly at $T^{*}=1.0$ ) even to $0\left(1 / T^{* 5}\right)$.

In conclusion, we have demonstrated that the numerical technique presented here is a viable method to compute the second virial coefficient of polyatomic molecules whose interaction is represented by the system sketched in figure 1 . More sophisticated techniques are listed in reference [8].

\section{References}

[1] Ely, J. F., Hanley, H. J. M., and Straty, G. C., J. Chem. Phys. 59, 842 (1973).

[2] Ely, J. F., and Hanley, H. J. M., Molecular Phys. 30, 565 (1975).

[3] Hanley, H. J. M., Nat. Bur. Stand. (U.S.), Tech. Note 693 (1977).

[4] Pople, J. A., Proc. Roy. Soc. A221, 498, 508 (1954); Buckingham, A. D. and Pople, J. A., Trans. Faraday Soc. 51, 1029 (1955).

[5] Klein, Max and Hanley, H. J. M., J. Chem. Phys. 53, 4722 (1970).

[6] Mason, E. A., and Spurling, T. H., The Virial Equation of State (Pergamon, New York, 1969).

[7] Hirschfelder, J. O., Curtiss, C. F., and Bird, R. B., Molecular Theory of Gases and Liquids (John Wiley, New York, 2nd Ed., 1964), page 212 and table II-A of the Appendix: Gallagher, J. S., and Klein, Max, J. Res Nat. Bur. Stand. (U.S.), 75A, (Phys. and Chem.) No. 337 (1971).

[8] For example, Steele and Sweet have developed a procedure to evaluate $B^{*}$ based on an expansion of the intermolecular potential in spherical harmonics, Sweet, J. R., and Steele, W. A., J. Chem. Phys. 50, 668 (1969). Direct numerical integration of the multidimensional virial expression, equation (2), is standard, e.g., by the Monte-Carlo technique. See Evan, D. J., and Watts, R. O., Molec. Phys. 29, 777 (1975); 31, 83, (1976); and Stroud, A. H., Calculation of Multiple Integrals (Prentice-Hall, New York, 1972). 